\title{
Analysis of Various Optimization Techniques in Machine Learning
}

\author{
Harpreet Kaur \\ Assistant Professor, CSE, Department, Chandigarh University, Gharuan, Punjab \\ (India)
}

Article History: Received: 11 January 2021; Accepted: 27 February 2021; Published online: 5 April 2021

\begin{abstract}
Optimization model the goal of solving a task in the paramount way providing the best results and it implies a best view point for solving query or we can also define optimization as a way of probing an another solution with the maximum cost efficient and utmost practicable performance under the required constraints, by increasing the preferred factor and reducing the unprefferable ones. This increase provides the utmost result or inference without any obstacles or complications. In this paper, various optimization techniques with respect to image processing are studied, where image processing deals with certain tasks and they are Image segmentation, image compression, image enhancement, image restoration (in painting).This paper provides basic knowledge of some optimization techniques.
\end{abstract}

Keywords: Image inpianting, Optimization techniques, Machine Learning

\section{Introduction}

Image rebuilding is an important phenomenon in the image methodology operation as it recompense for the defect which degrades the quality of image [1]. In the range of applications of computer vision, the image processing is usually known for the removal or reduction of noise or other distortion from the image which is captured by a digital camera or by another method of capturing image to provide a noise free and an enhanced image. This deprivation arises in many forms such as action haze, noise or if the camera is unable to focus. In cases of degradation which deals with the moving parts, it is feasible to move toward the high-quality estimation of the parameters which leads to removal of this blur/ haze effect in order to achieve the better quality [2]. There are certain cases where the quality of an image is reduced due to the presence of noise. The various category of noise are impulsive noise, Gaussian noise etc [3]. In the literature, various methods are found to remove the noise from an image such as Anisotropic diffusion, Dark frame subtraction, Non-local means, Wavelet transform and other filtering methods like converse filter, Weiner filter, Blind de-convolution, Wavelet re-establishment [4] The effective way of reducing the noise in an image is the use of various optimization techniques . In this paper, we have introduced certain optimization mechanisms used in processing of an image to get an optimal outcome that is used to restore original image. This paper provides detailed information of optimization techniques like Cuckoo search optimization, Grey wolf optimization (GWO), Grasshopper optimization algorithm (GOA) which can be used in dealing with images. These optimization algorithms provide better results which increases their importance in various applications of image processing namely biomedical application, spatial applications.

Various optimization techniques are discussed in section I, Literature survey is given in section II, Conclusion is provided in section III.

\section{Literature survey}

Anand Nayar and Dr.Rajeshwar Singh[51] have presented a computational Swarm intelligence technique along with its variants and to highlight the working of this technique in solving various discrete ,stochastic and dynamic problems. The techniques used by them are ACO,PSO,Fish Swarm which are gaining central point of attraction to various researchers around the nook.

He Zheng, Gang Yang, junyan Yi,Zheng Yuan,Xiaowei Liuand Meng Shuai [52] have presented basic rules to remove gratuitous behavioral rules from the fundamental Artificial bee colony technique, and investigated how uncomplicated the behavior regulations can be with no transform in presentation on frequency used benchmark. Firstly they have made analysis on the behavioural part and then recommended a clarify and easier version named as 'Simplest rules algorithm' for accounting the best performance of ABC algorithm.

James Kennedy and Russell Eberhart[28] have described the relationship between the various techniques like 'artificial bee colony', particle swarm optimization' and 'genetic algorithms'. In this the concept of fitness is used and according to authors particle swarm optimization technique serves in social and psychological way.

Seyedali Mirjalili,Seyed Mohammad Mirjalili and Andrew Lewis [53] have considered three classical engineering design problems and presented an authentic function of the planned scheme in the turf of 'optical engineering', the result to this shows that the projected technique is pertinent in testing the tribulations by unidentified places. The results show that this technique have soaring presentation in unidentified tough areas. 
Szymon Lukasik, Piortr A.Kowalski,Malgorzata Charytanwicz and Piotr Kulczycki [54] have presented an algorithm along with its experimental evaluation for a set of benchmark instances [55-58].Over a course of our study it was established that clustering in GOA is characterized by high accuracy when compared with K-means procedure.It has provided a new clustering mechanisms for better or improved value of factor c. possibly using lower bounds fixed values [59-62].

\section{Optimization algorithims}

\subsection{Ant colony optimization}

ACO be gotten because of the conduct of genuine ants for looking through nourishment. This technique was basically provided by 'Marco Dorigo' in 1992 to provide inferences for various discrete tribulations regarding optimization as well as unremitting tribulations. The major task for an ant is to bring food for its survival where the food is considered as the target and the method applied by the ant is considered as the 'optimization technique' in order to reach the food or target. In a real world scenario, ants move randomly for searching food and when they are able to discover the food or target then the next task is to return back to their own place which is named as 'Colony'. In order to ensure the path, these ants produce a liquid in their path named as 'Pheromone' and this process is known as 'pheromone trails'.

Pheromone trails is a chemical substrate or excreta which is released from the body of an individual (ant) that leads members of its own species towards a food source, without moving randomly, it provide a direct path or single path to other ants towards food [5].This technique is basically used to solve the 'computational' tribulations that can be abridged with the discovery of fine path from end to end through a chart.

Ant colony optimization was developed by Dorgis in 1991 [6, 7]. The first improvement in ACO known as Etilist Strategy for Ant System (EAS) was given by Dorgis.Im EAS, when the ants have discharged pheromone on the associations related to their produced solitions, the Daemon plays out an extra pheromone store on the edge to best arrangement found. Max Min Ant System(MAS) was created by Hoos in 1996 [8,9].It fundamentally constrains the pheromone trail value.The instatement of pheromone trail is finished by upper pheromone trail.By doing this, it will build the finding of arrangements at the beginning stage of search. Rank Based Ant System is an another improved adaptation of Ant System [10]. It was given by Bullnheimer et. al in 1997.The stored measure of pheromone legitimately relies upon ants rank and on the arrangements quality. Best Worth Ant System (BWAS) was purposed by Cordan et. al in 1999 [11,12].It don't co work with the customary computational ideas. BWAS utilizes it change rule just as pheromone dissipation system. What's more, to improve ants arrangement, it likewise takes the orderly abuse of nearby streamlining agents. Not just this, It likewise gives arrangement if pheromone trails gets struck by considering by re-instatement of pheromone trails. Amarjot kaur in 2017 played out the assessment of article location algo by utilizing subterranean insect settlement enhancement based picture segmentation[13].This paper presents an enormous assortment of techniques dependent on object identification and profound conviction networks. Wenbo Zhang et. al in 2018 proposed another calculation to illuminate multijoin inquiry enhancement dependent on equal ACO[14].The recreation result shows that equal ACO is more effecient and compelling. Yangping Wang et. al in 2018 improved the organization of ants and heuristic capacity to separate the total street information[15].The results shows that it can improve the nature of extricated streets by raising street's rightness.

ACO is basically an example of soft computing and other techniques can be artificial neural networks, genetic algorithms, and fuzzy logic. ACO assume a significant job for explaining vulnerabilities in picture handling incorporates clamor, fragmentation, incomplete and imprecise information. Special features of ACO are distributed and concurrent system, iterative system, search capabilities, colony based multi-agent approach. 


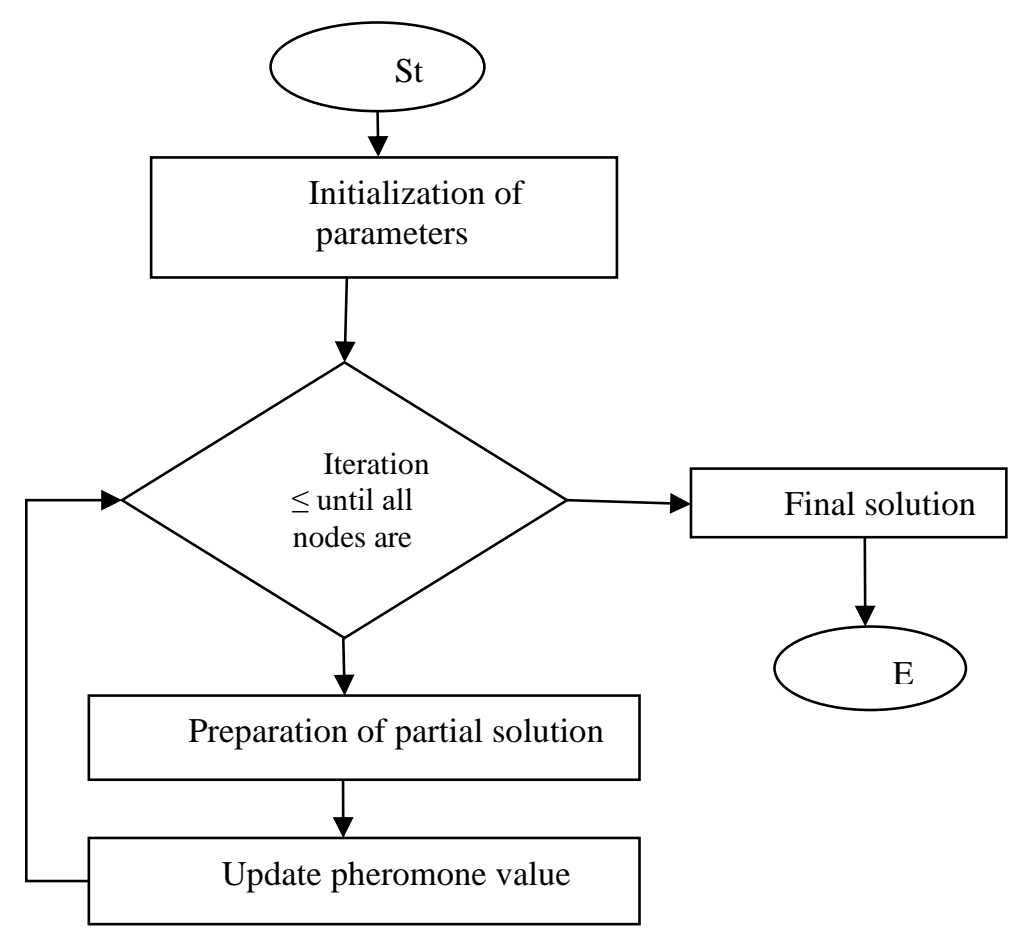

Pheromone Update:

Figure 1 Flow chart of ACO

$$
T x y \leftarrow(1-\rho) T x y+\sum_{k} \Delta T x y^{k}
$$

Where Txy, $\rho, \Delta$ Txyk are the quantity of stored pheromone for a state transition xy, pheromone evaporated coefficient and quantity of stored pheromone by kth ant respectively.

$\Delta T x y^{k}=\left[\begin{array}{c}\frac{Q}{L k} ; \text { if ant } k \text { uses curve } x y \text { in its tour } \\ 0 ; \text { otherwise }\end{array}\right]$

Where 'Lk' refers to the outlay of ' $k$ th ant's' tour and Q is stable.

\subsection{Counterfeit honey bee territory}

Counterfeit honey bee state territory or artificial bee colony (ABC) calculation is propelled by the keen conduct of bumble bees and it was created by Dervis karaboga in the time of 2005. This is a streamlining procedure that mimics the maturing conduct of bumble bees which is effectively applied in different empirical issues on earth. For the enhancement of numerical test works, this calculation can be effortlessly utilized [16].

The significant components of this calculation are:

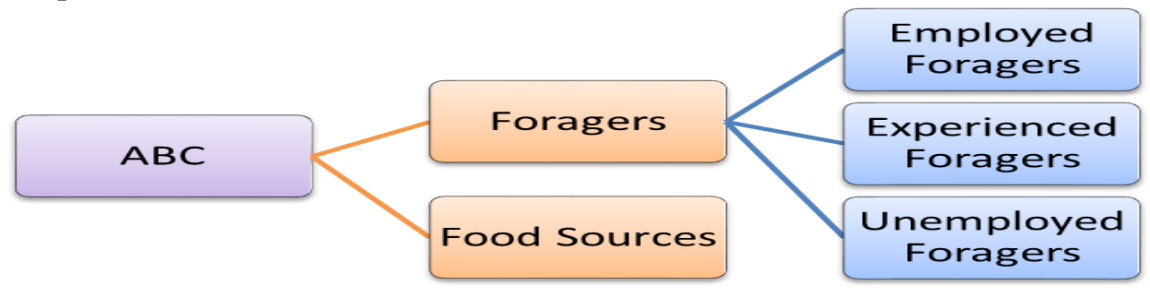

Figure 1 Elements of ABC algorithm

The mo significant object of the bees is to discover provision source by keeping in mind the important points like the amount of food with high nectar, distance from home and finally food source with highest nectar.

Utilized foragers are connected with a particular nourishment source which they are at present exploting. The fundamental elements of them are that they convey a data about that particular nourishment source, their separation and bearing from home, productivity of nourishment source and offer that data with lesser likelihood.

Jobless foragers continually search for a nourishment source. They are additionally partitioned into scouts which are in charge of scanning nature for new nourishment source encompassed by the nest, spectators sit in the home and discover nourishment sources with the assistance of data shared by utilized foragers. Correspondence 
among honey bees for trading the data about the nature of nourishment is the most significant marvel which happens in the moving locale and this move is called waggle move. Here spectators have enormous likelihood for the determination of productive and benefit making nourishment.

Artificial bee colony algorithm (ABC) was developed by Dervis Karaboga in 2007[17] for comprehending obliged streamlining problems. In his paper, he tested the performance of $\mathrm{ABC}$ on thirteen well known optimization problems and then compare it with PSO and DE(Differential Evolution).He concluded that ABC found the minimum of the seven of thirteen problems.From the results of mean,ABC is better than DE.Benala et. al in 2009 suggests that $\mathrm{ABC}$ algorithm can also be used for image enhancement[18].In this paper,the enhancement of the edges is done and the outcomes were analyzed with GA(Genetic Algorithm).Banharnsaken et. alsuggested the another version of $\mathrm{ABC}$ i.e. didtributed version and it was based on manager-worker problem[19].In his work,the process of communication as well as in synchronization is acheived through message transfering interface.Rashedi et. al also uses the ABC algorithm for solving the dynamic routing and wavelength allocated problem[20].The nature of the proposed algorithm based on dynamic routing was analyzed and the outcomes was better.Peng et. al suggested a modified extention of $\mathrm{ABC}$ algorithm[21].It concludes the best individual of the current itetration with the complete knowledge of global and food sources that lie at the best position. Junaedi et. al [22] compared his results with the results proposed by Asaju et. al.The suggest technique includes four Segments. The very 1st segment is based on placing subjects in the available space. The employee bee and the onlooker bee phase is based on searching rooms and place near the subjects by keeping in mind the minimization of constraint violations. Wu et. al introduced a calculation that depends on clusterung by utilizing honey bee's behaviour[23]. The bee's behaviour is used to predict function of unknown protein in protein to protein interaction network. The algorithm was stimulated in Matlab7.7, the outcomes were stimulated and then compared with other methods.Dongli et.al developed that ABC is the algorithm of swarm inteligence showing the nature of bees[24].The author also suggested the exchanging of neighbourhood during the search for food.Thhe results were more effecient and apeed had also been improved.Zhang et. al introduced the multiobjective $\mathrm{ABC}$ technique for solving QOS based routing problem[25].The existing bee behaviour had been differentiated with the proposed Genetic algorithm.Results were more attractive in terms of solution and performance. Zhang et. al suggested a co-evaluation model by integerating the multi-hive properties of honey bees with swarm intelligence[26].The stimulation of the experiment was done on five benchmark problems to compare the performance of both algorithms. So as to have a superior comprehension about the idea of assortment of nourishment (information), let us consider two nourishment sources X and Y. At first, the jobless honey bees start their work of looking through nourishment source. On the off chance that the jobless honey bee is a passerby, at that point it inclines toward the waggle move first and afterward after the assortment of information it continues. When it obtains food source the bee become employed solitary. After this, it starts discharging the nectar to its hive. Once the above process is completed, the bees enter to different zones. As bees does not return to same food source again so they become the Unemployed Follower. Before bees return to the same food source again, they enter into the area of information exchange and shares details with other bees (new bees). The honey bees don't come back to the waggle moving district yet keeps on chasing around a similar nourishment source. The means to be followed are given as follows:

1. Initialization of arrangement populace.

2. Send the scouts towards beginning nourishment sources

3. Repeat.

4. Send the utilized honey bees towards sources and gauge their nectar's sum.

5. Determine the real probability of sources with which they are progressively favored by spectators.

6. Send the spectators towards sources and gauge their nectar's sum.

7. Halt the abuse procedure of sources devoured by honey bees.

8. Send the scouts in new quest territory for finding new sources arbitrarily.

9. Memorize the best nourishment source found up until this point.

10. Until the prerequisites are met.

At first, the populace has some number of utilized honey bees, on looker honey bees and one scout honey bee. At the main stage an irregular quest is accomplished for the nourishment sources by the honey bees and the amount of nectar is assessed. In ABC calculation, there are sure cycles and each cycle comprises of three stages given beneath. The initial step manages the interchange of data between the utilized honey bees and the new honey bees and this procedure happens as the utilized honey bees come into hive and afterward the data is partaken in the moving region. The spectators get the data and search the most beneficial nourishment source. The utilized honey bee comes back to a similar nourishment source as its area is retained in the past cycle. In the event that the utilized honey bees experience nourishment source having most elevated nectar sum it retains another area and overlooks the old area. The subsequent advance arrangements with the determination of nourishment by the spectator honey bees, as they assess the measure of nectar and likelihood of choice is legitimately connected to the amount of source. The third step manages the substitution procedure. Right now, source is abandoned by the new nourishment source controlled by a scout honey bee. 


\subsection{Particle Swarm Optimization}

Particle swarm algorithm is occupants based advancement strategy and is presented by Dr.Eberhart and Dr.Kennedy in 1995, that is mixed by the conduct of fowl rushing. PSO is like some other improvement strategies, for example, 'Hereditary Algorithms'. Initially, this was produced for virtual products yet later on they understood that this calculation can be utilized in taking care of the advancement issues. This improvement calculation is very simple to place into activity. This technique depends on the set of iterations which are basically the variables obtained providing values closer to the result or target at a given moment where the random solutions and searches are used for getting the optimal solution. As genetic algorithm consists of two parameters named as crossover and mutation, these parameters are also known as evolution parameters [27]. But the particle swarm optimization algorithm does not contain these evolution parameters. Molecule swarm enhancement was created by James Kennedy and RC Eberhart in 1995[28].He portrayed the connections among Pso and both fake honey bee and hereditary algorithms.Particlw swarm worldview found the worldwide ideal each run and it surmised brings about terms of number of assessments required to arrive at certain performance stages.In 1997 Kennedy and Eberhart also presented the Binary PSO[29].In this algorithm,the position of particle is discete and velocity of particle is continous. Shi et. al in 1998 developed the standard PSO[30].This algorithm is generally usec to control the exploration and exploitation by inertia constant.He also improved the convergence quality of PSO by testing the performance on the different types of claasical function.Quyang A et. al in 2009 has presentes the another version called hybrid PSO [31]. With the help of this algorithm ,it is posible to solve non linear functions and best initial guess for simplex approach can also be find. L.m. Palanivelu in 2012 investigated the function of optimization for multiapplication smart card by applying image compression methods[32]. The segmentation of area of interest is investigated to get higher compression rate.The ROI and Non - ROI is compacted by utilizing lossless and lossy pressure individually. Molka Dhieb et. al in 2014 built up the 2-D entropy picture division on thresholding[33].In this paper,the creator introduced the two dimensional histogram investigation by entropy expansion The combination is improved and the proposed technique gets the perfect division result.Liny Li in 2013 proposes the Adaptive multi-scale division of high goals remote detecting images[34].In this paper,the writer constructed the particles in the swarm and swarm scan procedure is utilized for the division parameter selection. Based on PSO, multiscale division can get fulfilled outcomes. Mana Anantathanavit in 2013 recommends the Radius PSO(RPSO) [35].The creator proposed the RPSO so as to forestall the falling into nearby ideal. This strategy was tried in notable benchmark dataset and RPSO is better than conventional PSO in tackling the multimodal complex issues. Salim Lahmiri in 2016 introduced the joined incomplete differential equation sifting and PSO for uproarious biomedical picture segmentation [36].In this, the framework was tried on chest X-Ray picture which is obliterated with various degrees of Gaussian commotion. Shobith Narayan in 2018 presented the circularly polarized patch antenna by using pin loaded technique with PSO [37].The position of inductive pins was obtained by numerical approaches .The converged results from PSO satisfied the objective as well as design specifications of reflection coefficient.PSO contains probable resolution named as 'particles'. In this the difficulty is solved by the subsequent 'current particles' (link the sentences). This algorithm can be easily explained by taking into reference the flock of birds encompassing a region where they can smell the concealed nourishment. The flying creatures that arrive at nearest to the nourishment or target trills the most intense that gives a sign to different winged creatures for the bearing or way. Comparable procedure happens if another flying creature arrives at a local closer to the area of the past winged animal. This procedure proceeds until one of the flying creatures arrives at the objective which shows that PSO is anything but difficult to actualize. This calculation monitors the three factors which are 'Target value', 'Worldwide best', 'Stopping value'. The molecule contains the information having an answer, the 'speed esteem' which demonstrates the measure of progress to be done in information and the 'conceivable best' esteem showing the nearest esteem. The decision of arrangement parameters goes about as a noteworthy part for execution of the framework. The original PSO algorithm is affirmed by using the subsequent equations.

$V_{m d}=V_{m d}+\operatorname{clRand} 1()\left(P_{m d}-X_{m d}\right)+\mathrm{c} 2 \operatorname{Rand} 2()\left(P_{g d}-\mathrm{X}_{m d}\right.$

$X_{m d}=X_{m d}+V_{m d}$

In the above conditions $\mathrm{c} 1$ and $\mathrm{c} 2$ are the positive steady and 'Rand1' and 'Rand2' cause irregular number in the range from 0 and $1 . X \_m 1, X \_m 2 \ldots . . . . X \_(m d)$ speak to the particles and $P \_m 1, P \_m 2 \ldots \ldots . . P \_(m d)$ speak to the best suitable worth. The marker ' $\mathrm{g}$ ' has a place with best particles among occupants and $\mathrm{V}_{-} \mathrm{m} 1, \mathrm{~V} \_\mathrm{m} 2 \ldots \ldots$ ..V_(md )Represents the different speeds of the particles. Condition (1) portrays the speed modification over the procedure and condition (2) shows the refreshing of position for each fly molecule. On the off chance that the estimation of V_(md )is more noteworthy than the client particular limit Vmax this outcomes into the progressively modification of Vmax esteem 


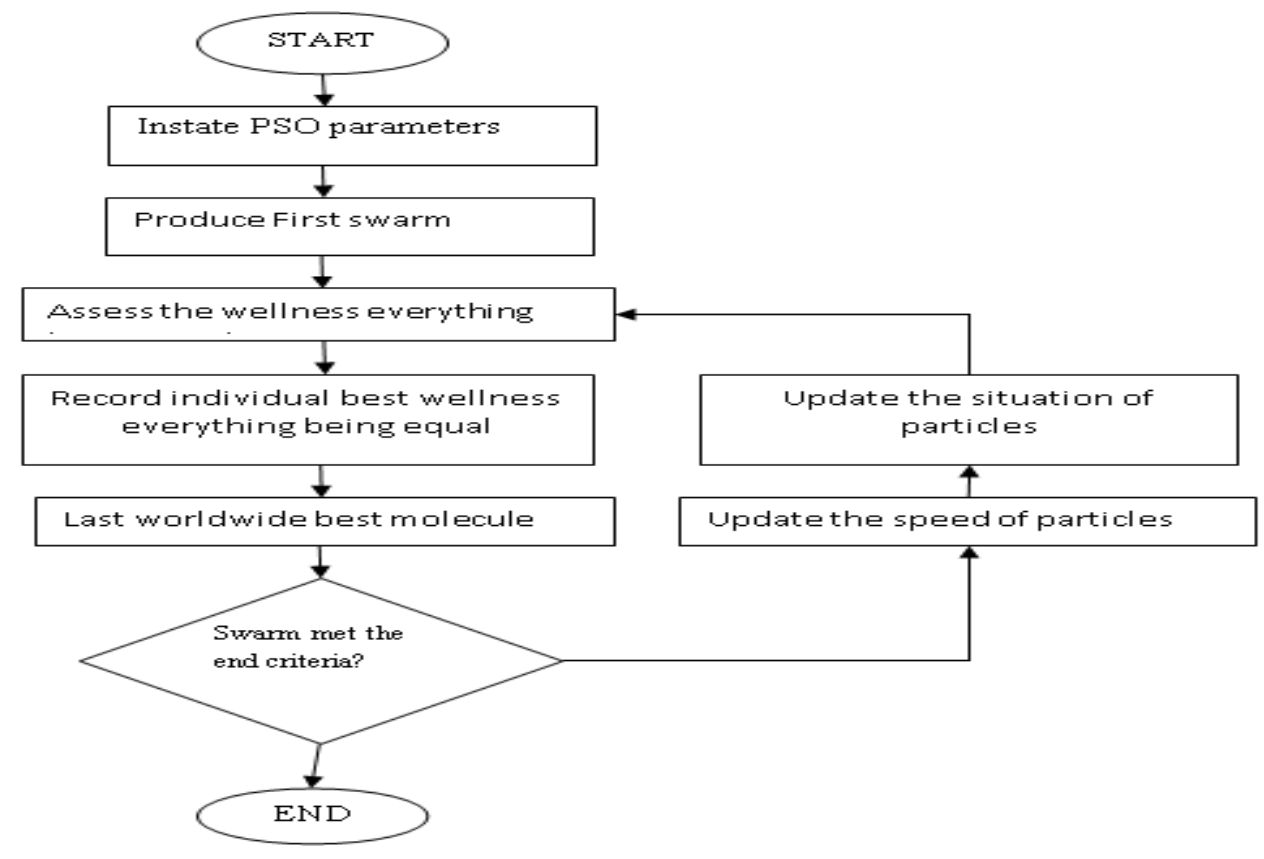

Figure 2 Standard flowchart of PSO

The steps used are specified below:

1. The first step is the initialization of particles.

2. Reiterate

3. Calculation of 'Best fitness 'value for each particle.

4. Compare both the values 'best fitness value' and 'p-best'.

5. Particle with the best fitness value is assigned to ' $\mathrm{g}$ '.

6. Vigorously make changes in the velocity and position according to the provided in both the equations.

7. Until the execution condition.

\subsection{Cuckoo Search Optimization}

Cuckoo search optimization is utilized in picture preparing is enrooted by Xin - She Yang and Suash Deb in 2009. This optimization calculation energized from the parasitic conduct of cuckoo winged animal, these feathered creatures have this regenerative system so as to expand their populace by laying their eggs in the homes of different fowls or species. For this conduct, cuckoo is otherwise called brood parasitic feathered creature. The cuckoo lays eggs in the homes of different winged creatures. In the event that the host flying creature discovers that the egg doesn't have a place with them then they toss the current egg or they leave the present home and make another home. Cuckoo search gives such rearing conduct which can be additionally applied in different enhancement issues and it performs better than other streamlining calculations in different utilizations of picture handling. In a nest, each one of the egg defines a elucidation and Cuckoo egg defines a latest and optimal solution of the existing problem. The resultant is the latest solution based on the existing one and amendment in some of its characteristics .In the simple terms each of the nest consists of an egg of cuckoo and rest of the eggs represent set of solutions Cuckoo search is effectively used to solve arrangement troubles and it is used to take care of improvement issue in structural engineering.

Following are the set of rules used in cuckoo search algorithm:

In the first rule is the selection of eggs, which takes place in random order and lays eggs in the selected nests.

The finest nests will be carried for the next creation.

The another rule is that for a set of nests the host bird can determine a distant egg with a likelihood pa $\epsilon$ $[0,1]$.The host Cuckoo can either chuck the egg away or discard the home and develop another elsewhere. The accompanying figure shows the stream outline of the cuckoo calculation

Levy flight: It is defined as a 'random walk', it is a kind of walk in which all the steps are associated with progression length, having a likelihood appropriation with the arbitrary rules. This random pace can also be seen in insects and animals. The first concern is the use of 'demand walk' and 'arbitrary stroll' in the standard condition to introduce most recent outcome.

$\mathrm{Xt}+1=\mathrm{Xt}+\mathrm{sEt}$

Where Et is taken from standard circulation having zero mean. These arbitrary strolls can be identified with the cuckoo egg and the host egg, which is confused in its execution

Yang and Deb in 2010 [38] has found another finding procedure utilizing Cuckoo Search in request to take care of building structure streamlining issues for example the plan of springs and welded bar structures. The essential goals of this method were to limit the heaviness of the spring and furthermore to limit the absolute manufacture 
cost. At that point, the outcomes were contrasted and other existing systems, for example, GA and PSO and it was demonstrated that Cuckoo Search is extremely effective and ends up being predominant for practically all the tried issues. What's more, Yildiz in 2012 [39] has utilized CS for choosing ideal parameters in processing operation.Chifu in 2012 [40] improved semantic web administration organization forms utilizing cuckoo search.

\subsection{Grey Wolf Optimization}

This algorithm was basically developed by MIRJALILI in the year of 2014 which is based on relationship between angling and ranking order of grey wolves. They are classified into alpha $(\alpha)$, beta $(\beta)$, delta $(\delta)$ and omega $(\omega)$. Not only this, but it also include three important steps of hunting consists of investigate victim, encircle around victim, attack on victim. Grey wolves are basically the active members who belong to apex predators i.e. their place is at the upper level of food chain. Their population generally tends to have a group called pack where the pack size is almost equal to 5-12 on average and their community ranking consist of four levels:

1. ALPHA $(\alpha)$ : Alpha is considered as a first level. The alpha ones can be male or female and basically the leaders of a pack. Alpha ones take the decisions such as hunting, amount of time taken for walking, place for sleeping. Group follows the decision of alpha ones and admits or accepts the alpha ones by holding their tales downwards. Alpha's are the dominant in the group.

2. BETA $(\beta)$ : Beta takes the second level. Beta wolves lend a helping hand to the alpha wolves for taking decisions. Beta wolves can be male or female. Beta ones takes place of alpha wolves when alpha's passes away or become mature. Beta wolves strengthen the alpha's orders throughout the group and provide reply back to $\alpha$ ones.

3. DELTA $(\delta)$ : Delta lie down in the third level. They acquire the decisions of $\alpha$ wolves and $\beta$ wolves and they dominate the omegas. Delta wolves are further divided into following categories:

(a) Scouts: Correctly inspection around the boundaries of sector is the main function of them and if danger exists then it will warn the whole group.

(b) Sentinels: Protection of pack which is a group is important task of them.

(c) Hunters: Supporting the $\alpha$ and $\beta$ in angling and providing feed to the group

(d) Caretakers: Responsibility of anamic and disabled wolves in the group is major task of caretakers.

4. Omega ( $\omega)$ : Omega holds the fourth level. Omega's follow the decisions of alpha's, beta's, delta's. Omega ones are not crucial and the last ones allowed to chew.

Grey wolf encircling victim: The numerical analysis of the encircling behaviour of the grey wolves is specified by the accompanying mathematical statement

$\mathrm{D}=|\mathrm{C} . \mathrm{Xp}(\mathrm{t})-\mathrm{A} \cdot \mathrm{X}(\mathrm{t})|$
$\mathrm{X}(\mathrm{t}+1)=\mathrm{Xp}(\mathrm{t})-\mathrm{A} \cdot \mathrm{D}$

Where $\mathrm{t}, \mathrm{A}, \mathrm{C}, \mathrm{Xp}$ and $\mathrm{X}$ are the current iteration, coefficient vectors, location vector of victim and location vector of grey wolf respectively.

Estimations of the vectors $\mathrm{A}$ and $\mathrm{C}$ are determined as:

$\mathrm{A}=2 \mathrm{a} \cdot \mathrm{r} 1-\mathrm{a}$

$\mathrm{C}=2 . \mathrm{r} 2$

Here segment of 'a' are directly diminished from 2 to 0 and r1,r2 are irregular vectors in $[0,1]$

Grey wolf hunting victim: Alpha wolves govern the hunting procedure. The main two categories of them i.e. alpha and beta takes participation in hunting occasionally .In the numerical analysis of angling, we assumed that $\alpha, \beta, \delta$ knows about the sector i.e. boundary of victim of and renew of the places of other hunt specialists(counting omega) depend on the situation of best inquiry operator. The scientific conditions are:
$\mathrm{D} \alpha=|\mathrm{C} 1 . \mathrm{X} \alpha-\mathrm{X}|$
$\mathrm{D} \beta=|\mathrm{C} 2 . \mathrm{X} \beta-\mathrm{X}|$
$\mathrm{D} \delta=|\mathrm{C} 3 . \mathrm{X} \delta-\mathrm{X}|$
$\mathrm{X} 1=\mathrm{X} \alpha-\mathrm{A} 1(\mathrm{D} \alpha)$
$\mathrm{X} 2=\mathrm{X} \beta-\mathrm{A} 2(\mathrm{D} \beta)$
$\mathrm{X} 3=\mathrm{X} \delta-\mathrm{A} 3(\mathrm{D} \delta)$
$\mathrm{X}(\mathrm{t}+1)=\mathrm{X} 1+\mathrm{X} 2+\mathrm{X} 3 / 3$

(1)

Ghazzai et.al in year 2014 proposed approach dependent on GWO so as to take care of cell arranging issues for 4G cell networks [41].The essential capacity of GWO is to discover the ideal base stations. But their tests proved that PSO beat GWO. Chaman-Motlagh in 2015 created structuring of photonic precious stone channels for looking through elite plans by GWO [42]. They tackled the issue as an improvement issues of single target function. as et. al in 2015 tested GWO for controlling rate of a second request DC motor systems[43]. Great transient reaction esteems were acquired when contrasted with PSO and ABC.Fouad et. al focused in 2015 , the confinement issue in WSN's[44].The creator built up a sink hub limitation approach dependent on GWO.The essential target was to look through the hubs with higher number of neighbors and their leftover energies are high.Chandr et. al in year 2016 applied the adjusted version of GWO dependent on six benchmark capacities for the choice of web administrations with great prerequisites of QOS[45].

Grey wolf attacking victim: The final process will be completed when it attacks on victim . The vector $\mathrm{A}$ is any arbitrary number lie in interval [-2a,2a] and a will go downwards from 2 to 0 . When $|\mathrm{A}|<1$, this is called a exploitation process in which the they attack on the prey i.e. convergence towards prey. 
Grey wolf searching victim: Creation of random population is a starting step of searching process. Alpha, Beta, Delta calculate the exact area of prey and update its distance from prey. When $|\mathrm{A}|>1$, this is called exploration process i.e. divergence from prey.

The algorithm to be used is specified below:

1. Population Initialization $\mathrm{XK}(\mathrm{K}=1,2,3, \ldots, \ldots, 1)$

2. Initialize A, C and a

3. Choose source and sink arbitrarily

4. Evaluate number of clusters according to mathematical statements given above

5. Evaluate number of centeroids

6. Evaluate the fitness for each wolf XK

7. Raise clusters functions

8. Store the most limited way from source to goal

9. Set the estimation of the limit C $(I, j)$

10. Raise maximum flow function

11. Return the optimum solution

\subsection{Grasshopper Optimization}

GOA is an algorithm which was developed by MIRJALILI . that reveals behavior of grasshopper and their social interaction. Grasshoppers are responsible for the destruction of agriculture due to their destructive behaviour. In the existence pattern of grasshopper, there are two stages known as NYMPH and ADULTHOOD. The NYMPH grasshopper doesn't have wings, so they move gradually and eat all vegetation on their way. At the point when they grow up and reach at the adulthood with wings to shape a swarm in air and move quick. Grasshopper enhancement calculation is a populace based, in which every grasshopper speaks to an answer in the populace. The three powers on which position of every grasshopper depends are the social communication among individual and other grasshopper 'Si', gravity power on grasshopper 'Gi', wind cooperation 'Wi'.

The last type of three influenced powers on every grasshopper can be given as:

$\mathrm{Xi}=\mathrm{Si}+\mathrm{Gi}+\mathrm{Ai}$

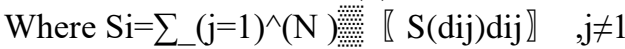

$\mathrm{S}$ is characterized as the capacity that denote the quality of two social power, fascination and repugnance between grasshopper

$\mathrm{Gi}=-$ geg

Where $\mathrm{g}$ is the gravitational consistent, model is a focal point of earth solidarity vector.

$\mathrm{Ai}=\mu \mathrm{ew}$

Where $\mu$ is consistent float and ew=wind heading solidarity vector

Simpson SJ et.al [46] gave a survey on this procedure that despite the fact that grasshopper are generally observed exclusively in nature, they participate in one of the biggest swarm of all creatures.Rogers SM et. al [47]investigated the novel part of grasshopper swarm for example the swarming nature lie in both sprite and adulthood. Anas Atef Amaireh et. al in 2017 structured a direct radio wire cluster by utilizing antlion and grasshopper optimization[48].They are utilized to reducw the side projection by upgrading the excitation current amplitudes of exhibit components. Neelam Rajput in 2017 introduced an ideal age booking of warm framework utilizing organically roused grasshopper algorithm[49].This paper illuminate three distinct sorts of ED issues comprising little, medium and enormous scope power framework with various intricacy levels. The execution of GOA is very prevalent for tackling ED issues. Baran Hekimoglu in 2018 proposed a GOA for automatic voltage regulation syatem[50].The simplicity of this algorithm gives a high quality tunung of optimal PID(proportional integral derivative) controller parameters.

The algorithm used is specified below:

1. Initialization

2. Initial population and evaluation

3. Assigning the overall best solution

4. Updating the decreasing coefficient parameter

5. Mapping the distance or grasshoppers

6. Updating solution

7. Solution boundaries voilation

8. Visiting all the solutions in population

9. Solutions evaluation

10. Termination criteria

11. Returning the best solution

\section{Conclusion}

This article comprises of the study of certain optimization algorithms which can be further used in the areas of digital image processing like restoration, segmentation, compression, and enhancement. Instead of a variety of existing 'image in painting' techniques, optimization techniques are extensively used by numerous researchers to 
acquire the finest restored or unpainted image having negligible loss of data. Other favorable advantage of these systems is that these strategies likewise limit an opportunity to reestablish an image from the noisy picture without relinquishing the nature of an image.

\section{References}

A. Quyang, Nanning, Y. Zhou and Q. Luo, "Hybrid particle swarm optimization algorithm for solving systems of nonlinear equations", " Proceeding in IEEE International Conference on Granular Computing”, ISBN 978-1-4244-4830-2, pp. 460 - 465, 2009

1. A.Townsend, "Genetic Algorithm-a Tutorial" ,2003.

2. Amarjot Kaur,Navleen Kaur, "Performance Evaluation of Object Detection Algorithm using Ant Colony Optimization Based Image Segmentation", "IEEE International Conference on Computing Communication,Control and Automation",2017.

3. Anand Nayyar and Dr.Rajeshwar Singh, "Ant Colony optimization- computational swarm intelligence technique ", “ InternationalConference on Computing for Sustainable Global Development (INDIACom)", 2016.

4. Anas Atef Amaireh,Asem Alzoudi,Nihad, "Design of Linear Antenna Array using Antlion and Grasshopper Optimization Algorithms", "IEEE Jordan Conference on Applied Electrical Engineering and Computing Technologies”,2017.

B. Bullnheimer and C. Strauss, "A New rank-based version of the Ant System: A computational study," Central Joournal for Operations Research and Economics, vol. 7, pp.25-38, 1999.

5. Banharnsakun, Anan, Tiranee Achalakul, Booncharoen Sirinaovakul, "Artificial bee colony algorithm on distributed environments", "Nature and Biologically Inspired Computing (NaBIC), Second World Congress, IEEE", 2010, pp. 13-18.

6. Baran Hekimoglu,Serdar Ekinci, "Grasshopper optimization algorithm for automatic voltage regulator system", "IEEE 5th International Conference on Electrical and Electronic Engineering”,2018.

7. Batoo, K. M., Kumar, G., Yang, Y., Al-Douri, Y., Singh, M., Jotania, R. B., \& Imran, A. (2017). Structural, morphological and electrical properties of $\mathrm{Cd} 2+$ doped $\mathrm{MgFe} 2-\mathrm{xO} 4$ ferrite nanoparticles. Journal of Alloys and Compounds, 726, 179-186.

8. Batyrkhan Sultanovich Omarov et.al , "Exploring Image Procesing and Image Restoration Techniques", "International Journal of Fuzzy Logic Intell System", 2015.

9. Benala Tirimula Rao et. al, "A Novel Approach to Image Edge Enhancement using Artificial Bee Colony Algorithm for Hybridizwd Smmoothing Filters" , "Nature and Biologically Inspired Computing”,2009.

10. Chaman-Motlagh A, "Superdefect photonic crystal filter optimization using grey wolf optimizer", “ IEEE Photonics Technol Lett 27(22):2355-2358”,2015.

11. Chandra M, Agrawal A, Kishor A, Niyogi R, "Web service selection with global constraints using modified gray wolf optimizer" IEEE international conference on advances in computing, communications and informatics (ICACCI)", pp 1989-1994,2016.

12. Chifu, V, Pop, C,Salomie, I, Suia, D, Niculici, A, “Optimizing the Semantic Web Service Composition Process Using Cuckoo Search. Intelligent Distributed Computing”,2012.

13. Das KR, Das D, Das J, “ Optimal tuning of pid controller using gwo algorithm for speed control in dc motor", " IEEE International conference on soft computing techniques and implementations (ICSCTI)", pp 108-112,2015.

14. Dervis Karaboga ,Bahriyre Bastruk, "Artificial Bee Colony Optimization Algorithm for Solving Constrained Optimization Problems", "12th Foundations of Fuzzy Logic and Soft Computing ,Cancun,Mexico",2007.

15. Dongli, Zhang, G. Xinping, T. Yinggan, T. Yong, "An artificial bee colony optimization algorithm based on multiexchange neighborhood", Computational and Information Sciences (ICCIS), Fourth International Conference, IEEE, 2012, pp. 211-214.

16. Fouad MM, Hafez AI, Hassanien AE, Snasel, "Grey wolves optimizer-based localization approach in WSNs", “IEEE 11th international computer engineering conference (ICENCO)”, pp 256-260,2015.

17. Gairola, P., Gairola, S. P., Kumar, V., Singh, K., \& Dhawan, S. K. (2016). Barium ferrite and graphite integrated with polyaniline as effective shield against electromagnetic interference. Synthetic Metals, 221, 326-331.

18. Garg, S., Singh, A., Kaur, K., Aujla, G. S., Batra, S., Kumar, N., \& Obaidat, M. S. (2019). Edge computingbased security framework for big data analytics in VANETs. IEEE Network, 33(2), 72-81.

19. Ghazzai H, Yaacoub E, Alouini MS , "Optimized lte cell planning for multiple user density subareas using meta-heuristic algorithms”, “IEEE 80th vehicular technology conference (VTC2014-Fall)”,2014.

20. Guo, Peng, Wenming Cheng, Jian Liang, "Global artificial bee colony search algorithm for numerical function optimization", Natural Computation (ICNC), Seventh International Conference on. Vol. 3, IEEE, 2011, pp. 1280-1283. 
21. Gupta, M. K., Mia, M., Singh, G., Pimenov, D. Y., Sarikaya, M., \& Sharma, V. S. (2019). Hybrid coolinglubrication strategies to improve surface topography and tool wear in sustainable turning of Al 7075-T6 alloy. The International Journal of Advanced Manufacturing Technology, 101(1-4), 55-69.

22. He Zheng, Gang Yang, junyan Yi,Zheng Yuan,Xiaowei Liuand Meng Shuai , "Simplify the Basic Artificial Bee Colony Algorithm , " IEEE International Conference on Systems, Man and Cybernetics", 2015.

23. J. Kennedy \& R.C. Eberhart, “A discrete binary version of the particle swarm algorithm”, "In Proceedings of the IEEE International Conference on Systems, Man and Cybernetics, IEEE Press, Piscataway", NJ, 41044108, 1997.

24. J. Kennedy \& R.C. Eberhart, "Particle swarm optimization", “ Proceeding of IEEE International Conference on Neural Networks, Piscataway”, NJ., 1942-1948, 1995.

25. J.J Liang et. Al, "Novel Composition Test Functions for Numerical Global Optimization”, "IEEE Swarm Intelligence Symposium”, 2005.

26. Jindal, A., Aujla, G. S., \& Kumar, N. (2019). SURVIVOR: A blockchain based edge-as-a-service framework for secure energy trading in SDN-enabled vehicle-to-grid environment. Computer Networks, $153,36-48$.

27. Journal of Mathematical Modelling and Numerical Optimisation”,2010.

28. Junaedi, Danang, Nur Ulfa Maulidevi, "Solving Curriculum-Based Course Timetabling Problem with Artificial Bee Colony Algorithm", Informatics and Computational Intelligence (ICI), First International Conference, IEEE, 2011, pp. 112-117.

29. Kaur, D., Aujla, G. S., Kumar, N., Zomaya, A. Y., Perera, C., \& Ranjan, R. (2018). Tensor-based big data management scheme for dimensionality reduction problem in smart grid systems: SDN perspective. IEEE Transactions on Knowledge and Data Engineering, 30(10), 1985-1998.

30. L.M. Palanivelu and P.Vijayakumar,"A Particle Swarm Optimization for Image Segmentation in Multi Application Smart Cards", "European Journal of Scientific Research", ISSN 1450-216X, vol. 70, no. 3, 2012.

31. Liny Li, “Adaptive Multiscale Segmentation of High Resolution Remote Sensing Images Based on Particle Swarm Optimization", "IEEE 5th International Conference on Intelligent Human Machine Systems and Cybernetics",2013.

32. M. Dorigo, V. Maniezzo, and A. Colony, "The Ant System: Optimization by a colony of cooperating agents," IEEE transcations on Systems, Man, and Cybernetics, vol. 26, pp. 2941, 1996.

33. M.Dorigo and T. Stutzle, "Ant Colony Optimization,” MIT Press, Cambridge, MA, 2004.

34. Mana Anantathanavit,Mud-Armeen Munlin, "Radius Particle Swarm Optimization”, "IEEE International Computer Science and Engineering Conference", 2013.

35. Mittal, N., Singh, U., \& Sohi, B. S. (2017). A novel energy efficient stable clustering approach for wireless sensor networks. Wireless Personal Communications, 95(3), 2947-2971.

36. Mohd. Junedul Haque, “A Brief Review of Image Restoration Techniques" , "Journal of Advanced Computing Research”,2014.

37. Molka Dhieb et. al, "2-D entropy image segmentation on thresholding based on particle swarm optimization", "IEEE 1st International Conference on Advanced Technologies for Signal and Image Processing”,2014.

38. MS Munira A Jiwan,Mr. S.N.Dandare, "Single Image Fog Removal using Depth Estimation based on Blur Estimation" , "International Journal of Scientific and Research" ,2013.

39. Neelam Rajput et. al, "Optimal Generation Scheduling of Thermal System using Biologically Inspired Grasshopper Algorithms", 'IEEE 2nd International Conference on Telecommunication and Networks",2017.

40. Oscar Cordon and T. Stutzle, "A Review on the Ant Colony Optimization Metaheuristic: Basis, Models and New Trends," Mathware and Soft Computing, vol. 9, 2002.

41. Oscar Cordon, I. Frandez and L. Moreno, "A new ACO model integrating evolutionary computation concepts: the best-worst Ant System," Proceedings of ANTS2000, pp.22-29, Belgium, 2000.

42. Priyanka Kamboj,Versha Rani, "A Brief Study of Various Noise Model and Filtering Technique", "Jornal of Global Research in Computer Science" ,2013.

43. Rashedi, Arash, Yousef S. Kavian, K. Ansari-Asl, Z. Ghassemlooy, "Dynamic routing and wavelength assignment: Artificial bee colony optimization", "Transparent Optical Networks (ICTON), 13th International Conference, IEEE", 2011, pp. 1-4.

44. Rogers SM et. al, "Mechanosensory-induced Behavioural Gregarization in the Desert Locust Schistocerra Gregaria. J Exp Biol 2003;206;3991-4002".

45. Salima Lahmiri,Mounir Boukadoum, "Combined Partial Differential Equation Filtering and Particle Swarm Optimization for Noisy Biomedica Image Segmentation”, "IEEE 7 th Latin American Symposium on Circuits and Systems",2016. 
46. Seyedali Mirjalili,Seyed Mohammad Mirjalili and Andrew Lewis , "Grey Wolf Optimizer, semanticscholar.org".

47. Sharma, G., Sharma, S., \& Gujral, S. (2015). A novel way of assessing software bug severity using dictionary of critical terms. Procedia Computer Science, 70, 632-639.

48. Shobith Narayanan et. al, "A Circularly-polarized Patch Antenna using Pin-loaded Technique with PSO" , "International Conference on Advances in Computing, Communications and Informatic",2018.

49. Simpson SJ,McCaffery A,HAeGELE BF, "A Behavioural Analysis of Phase Change in the Desert Locust,Biol Rev 1999;74;461-80”.

50. Szymon Lukasik, Piortr A.Kowalski,Malgorzata Charytanwicz and Piotr Kulczycki, "Data Clustering with Grasshopper Optimization Algorithm ," "Conference on Computer Science and Information Systems" ,vol.11, pp. 71-74, 2017.

51. T. Stutzle and H. H. Hoos, "MAX-MIN Ant System," Future Generation Computer Systems, vol. 16, pp.889-914, 2000.

52. T. Stutzle and H. H. Hoos, "The MAX-MIN Ant System and local search for the travelling salesman problem," IEEE International Conference on Evolutionary Computation, pp. 309-314, Piscataway, 1997.

53. Tomer J Czaczkes et. Al, "Trail Pheromones:An Integrative View of Their Role in social Insect Colony Organization, “Annual Review of Entomology”,2014

54. Wenbo Zheng et. Al, "Database Query Optimization based on Parallel ACO” , "IEEE 3rd International Conference on Image ,Vision and Computing”, 2018.

55. Wu, Shuang, Xiujuan Lei, Jianfang Tian, "Clustering PPI network based on functional flow model through artificial bee colony algorithm", Natural Computation (ICNC), Seventh International Conference on. Vol. 1, IEEE, 2011, pp. 92-96.

56. Y. Shi \& R.C.Eberhart, “A Modified Particle Swarm Optimizer”, “In Proceedings of the IEEE Congress on Evolutionary Computation", 1998.

57. Yang, X. S, Deb, "Engineering optimisation by cuckoo search”, “International

58. Yangping Wang et. Al, "Road Extraction from High Resolution Remotely Sensed Image Based on Improved Ant Colony Optimization” ,IEEE International Conference on Dependable,Automatic and Secure Computing",2018.

59. Yildiz, A. R, "Cuckoo search algorithm for the selection of optimal machining parameters in milling operations", "The International Journal of Advanced Manufacturing Technology”,2012.

60. Zhang, Changsheng, Y. Li, Z. Li, B. Zhang, "A multi-objective artificial bee colony algorithm for QoS based route optimization problem", Systems and Informatics (ICSAI), International Conference, IEEE, 2012, pp. 1538-1541.

61. Zhang, Hao, Yunlong Zhu, Xiaohui Yan, "Multi-hive artificial bee colony algorithm for constrained multiobjective optimization", Evolutionary Computation (CEC), IEEE, 2012, pp. 1-8. 